\title{
Polyarthritis, fever and a rash in a young girl
}

\author{
Katherine Matheson $\mathrm{BSC}^{1}$, Joanne M Langley MD MSc FRCPC ${ }^{2,3}$, \\ Bianca Lang MD FRCPC ${ }^{2}$, Timothy Mailman MD FRCPC $2,4,5$
}

\begin{abstract}
A previously healthy six-year-old girl had developed a fever Afive days before presentation. Two days later, she had an onset of diffuse abdominal pain, intermittent vomiting and loose, nonbloody stools. On the fourth day of fever, she described pain on moving her arms and legs, and the following day, she was brought to the IWK Health Centre in Halifax, Nova Scotia. Her history showed up-to-date immunizations, and the absence of medications or known allergies. There was no history of trauma, infectious contacts, travel outside the Maritime provinces, or consumption of unpasteurized dairy products or undercooked meats. She had no history of animal exposure, except for a white rat kept in a cage in the family basement.

On examination, she was febrile $\left(38.8^{\circ} \mathrm{C}\right)$, unwell looking and tachycardic, but was oriented, normotensive and in no respiratory distress. Her head and neck examinations revealed periorbital edema, marked erythema of the buccal mucosa, and a 'strawberry tongue' with no oral ulcers, tonsillar exudate, cervical adenopathy or conjunctival injection. A blanching erythematous maculopapular rash was visible on the face, trunk, arms and legs, being most prominent on the hands and feet. A palpable, nonblanching petechial exanthem was also present on the palms of the hands and soles of the feet, with marked
\end{abstract}

periungual erythema and capillary vessel dilation at the nail beds.

No puncture marks or scratches were visible. The wrists, elbows, knees and small joints of the hands were swollen and warm to palpation, but without erythema. Any motion of these joints was limited and painful. There was periarticular swelling of the ankles and feet. The precordium was hyperdynamic, with a 2/6 vibratory systolic ejection murmur at the upper left sternal border. There were no bruits. There was no hepatosplenomegaly or generalized adenopathy.

The white blood cell count was $6.0 \times 10^{9} / \mathrm{L} \quad(56 \%$ neutrophils, $12 \%$ bands and $13 \%$ lymphocytes), the hemoglobin level was normal and the platelet count was $31 \times 10^{9} / \mathrm{L}$. The C-reactive protein level and erythrocyte sedimentation rate were elevated at $219 \mathrm{mg} / \mathrm{L}$ (reference less than $5.3 \mathrm{mg} / \mathrm{L}$ ) and $65 \mathrm{~mm} / \mathrm{h}$ (reference $0 \mathrm{~mm} / \mathrm{h}$ to $9 \mathrm{~mm} / \mathrm{h}$ ), respectively. Renal function and liver enzymes tests were normal. Urinalysis revealed trace blood, and hyaline and granular casts. Her chest radiograph and echocardiogram were normal.

Empirical antibiotic therapy was started. A blood culture taken on the day of presentation was positive at $15 \mathrm{~h}$.

What is the diagnosis?

\footnotetext{
${ }^{1}$ Faculty of Medicine; ${ }^{2}$ Department of Pediatrics; ${ }^{3}$ Department of Community Health and Epidemiology; ${ }^{4}$ Department of Pathology; Department of Microbiology and Immunology, Dalhousie University, Halifax, Nova Scotia

Correspondence: Dr Joanne M Langley, IWK Health Centre, 5850 University Avenue, PO Box 9700, Halifax, Nova Scotia B3K 6 R8.

Telephone 902-470-8498, fax 902-470-7232, e-mail Joanne.Langley@dal.ca

Received and accepted for publication October 19, 2007
} 


\section{DIAGNOSIS}

Fifteen hours after presentation, a pleomorphic, Gramnegative rod in chains was isolated from blood culture (BacT/ALERT, Organon Teknika, USA). Subculture produced growth on sheep blood agar only (no growth on MacConkey agar). Colonies were small, round and smooth, and had negative reactions for both catalase and oxidase. Growth in thioglycollate supplemented with horse serum yielded small 'puff balls' near the bottom of the tube. Although inert on standard biochemical tests, horse serum-supplemented tests were positive for esculin hydrolysis, hydrogen sulfide production, and acid production from glucose, maltose and mannose. Supplemented tests were negative for nitrate reduction, indole and urease, leading to the organism's identification as Streptobacillus moniliformis. Serology for Epstein-Barr virus, Parvovirus B19, Coxiella burnetii, Rickettsia rickettsii, Rickettsia typhi, adenovirus and syphilis was negative.

\section{DISCUSSION}

Rat-bite fever is a zoonosis caused by $S$ moniliformis or Spirillum minus, organisms found as commensal oral or nasopharyngeal flora in rats (1). A history of all animal exposures by the child during the infectious disease consultation revealed this clue that helped in the diagnosis. Although a bite is usually reported, rat-bite fever occurs following scratches, handling of rodents, or contact with their feces or urine in approximately $30 \%$ of cases (1). Our patient reported only handling of the rat.

While rat-bite fever refers to infection resulting from animal exposure, ingestion of this pathogen in food or water contaminated with rodent feces results in 'Haverhill fever', named for an outbreak associated with $S$ moniliformis-contaminated milk in Haverhill, Maine, USA, in 1926 (2). S minus is a noncultivatable, Gram-negative, spiral-shaped bacterium that is more commonly reported in Asia, where rat-bite fever is known as 'Soduku' (1). With either pathogen, an incubation period of three to 10 days precedes the onset of fever, chills, headache, vomiting, myalgias and rash. The rash may be maculopapular, petechial, vesicular or pustular; is most prominent on the extremities; and involves palmar and plantar surfaces. Arthritis is most common in the knees, but can affect ankles, elbows, shoulders and hips. Complications include endocarditis, myocarditis, meningitis, pneumonia, solid-organ abscesses and death in up to $13 \%$ of cases (1) .

Penicillin is the treatment of choice for S moniliformis infection, although the organism is also usually susceptible in vitro to cephalosporins, carbapenams, clindamycin, tetracycline and vancomycin (1). Intravenous treatment is given for at least seven days.

The differential diagnosis of fever, arthralgia and rash involving the extremities is broad (3). Viral infections such as the Epstein-Barr virus or Coxsackie virus B, as well as bacterial infections including meningococcemia, streptococcal infection, Lyme disease, leptospirosis, brucellosis, disseminated gonorrhea and other causes of sepsis syndrome, should be considered when faced with such symptoms. Rash on the palms and soles, which is often present in rat-bite fever patients, is also a symptom of Rocky Mountain spotted fever and secondary syphilis. The polyarthritic symptoms of rat-bite fever are similar to those of disseminated gonococcal infection, Lyme disease, brucellosis, septic arthritis, infective endocarditis, collagen vascular disease and acute rheumatic fever. If relapsing fever is present, Borrelia recurrentis, malaria and typhoid fever should also be considered in the differential. A careful history, consideration of local epidemiology and the age of the patient can assist with limiting these possibilities. Diagnosis is usually made by blood culture, although $S$ moniliformis is a fastidious organism that needs a microaerophilic environment and may take up to seven days to grow (1). Polymerase chain reaction has been used in culture-negative cases (4).

\section{REFERENCES}

1. Elliott SP. Rat bite fever and Streptobacillus moniliformis. Clin Microbiol Rev 2007;20:13-22.

2. Pilsworth R. Haverhill fever. Lancet 1983;2:336-7.

3. Hambidge SJ, Ogle JW. Index of suspicion. Case 1. Diagnosis: Rat-bite fever. Pediatr Rev 2001;22:95-103.

4. Berger C, Altwegg M, Meyer A, Nadal D. Broad range polymerase chain reaction for diagnosis of rat-bite fever caused by Streptobacillus moniliformis. Pediatr Infect Dis J 2001;20:1181-2. 


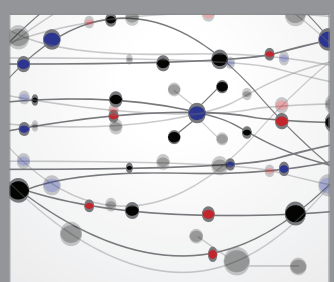

The Scientific World Journal
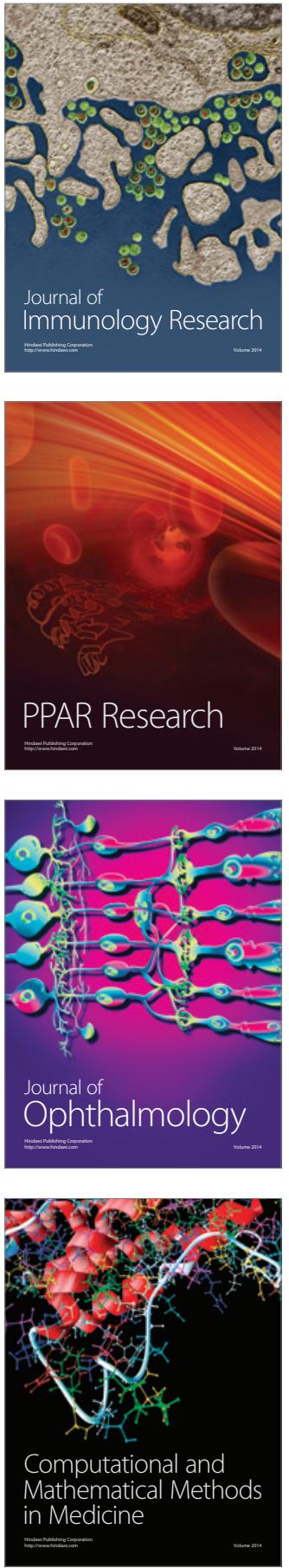

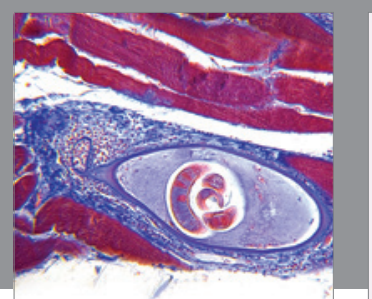

Gastroenterology Research and Practice

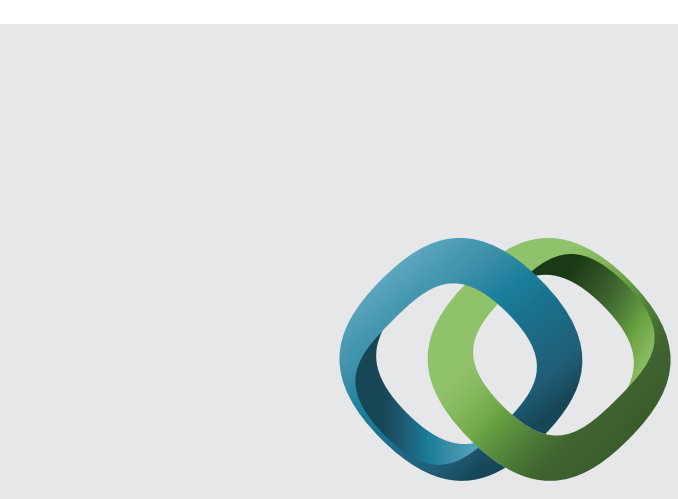

\section{Hindawi}

Submit your manuscripts at

http://www.hindawi.com
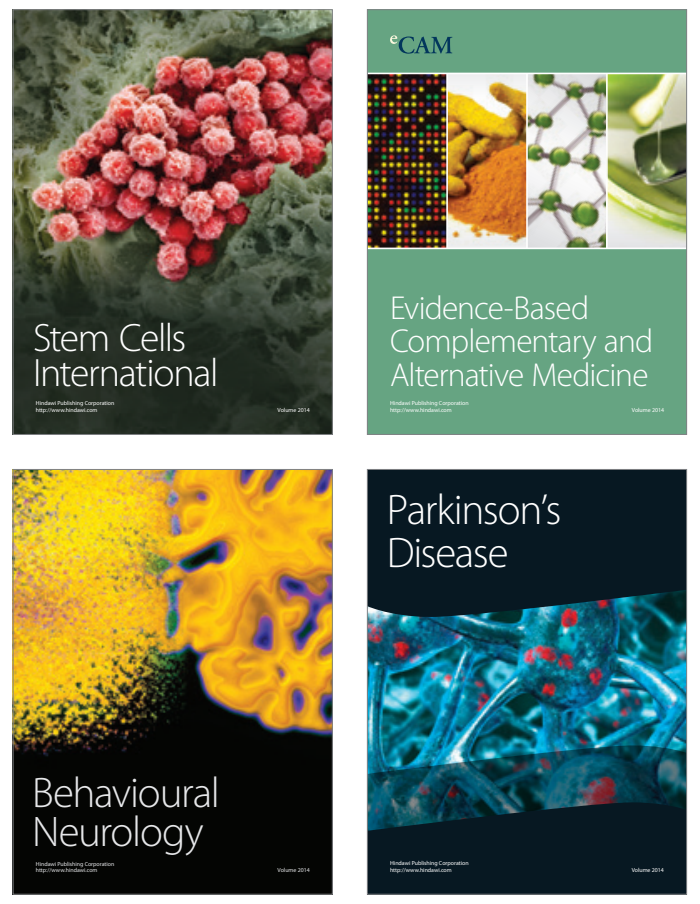
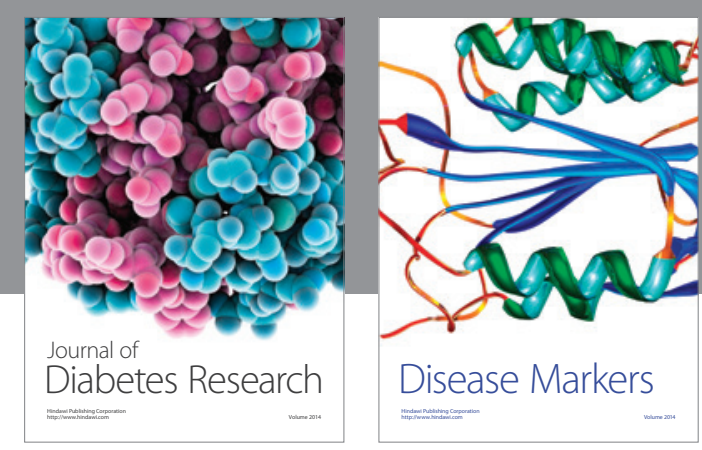

Disease Markers
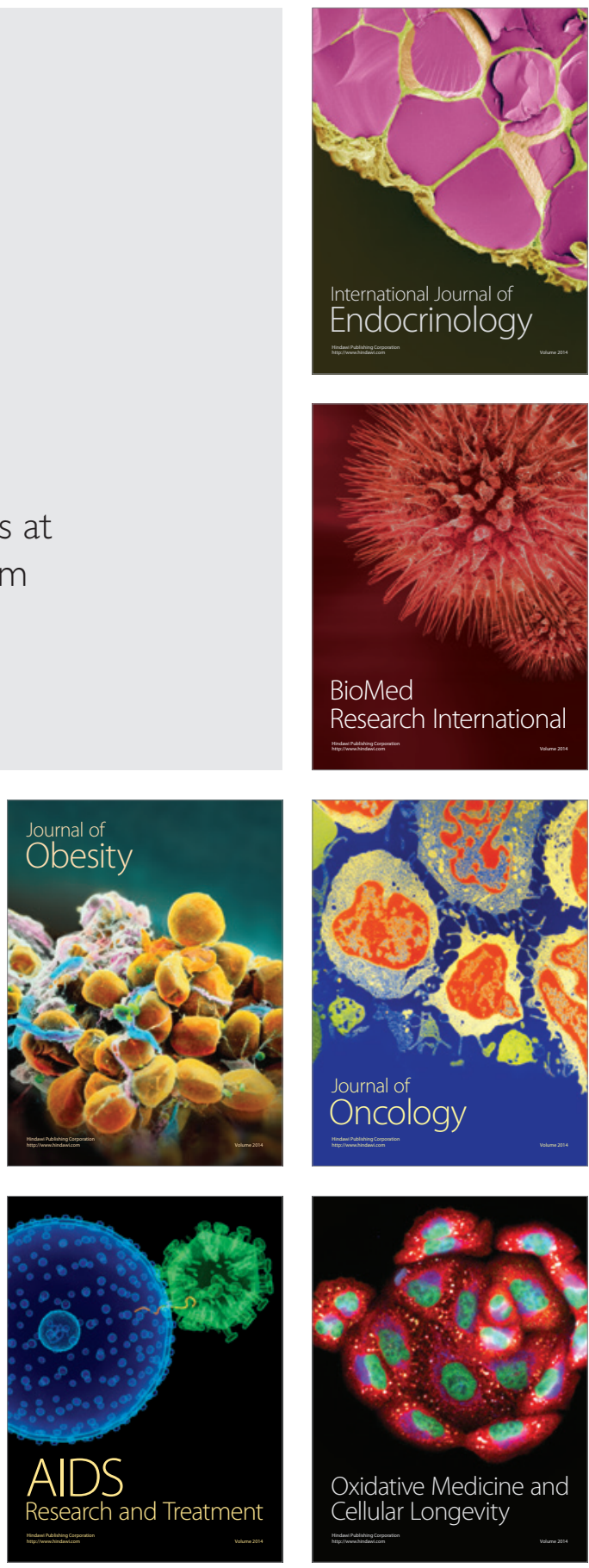